\title{
Echocardiographic abnormalities in type IV mucopolysaccharidosis
}

\begin{abstract}
Cardiac involvement is well recognised in most forms of the mucopolysaccharidoses but there is poor documentation of abnormalities specific to Morquio's syndrome (type IV mucopolysaccharidosis). Ten patients with the classic form or type A Morquio's syndrome with a median age of 12.5 years underwent echocardiographic assessment. Abnormalities were detected in six $(60 \%)$ cases with mitral valve involvement in five patients and aortic valve disease in four. One patient had severe mitral leaflet thickening to the point of mitral stenosis. Two patients had evidence for myocardial involvement by way of echocardiographic ventricular hypertrophy. The cardiac lesions were haemodynamically mild. A cardiac murmur was audible in only three of the six cases. It is concluded that there is a high prevalence of silent cardiac abnormalities in patients with Morquio's syndrome with predominantly left sided valve involvement. Echocardiography should be part of the assessment of these patients and bacterial endocarditis prophylaxis should be advised for those with cardiac abnormalities.
\end{abstract}

The mucopolysaccharidoses are one class of lysosomal storage disease produced by an inherited absence or defective activity of one of the enzymes involved in the degradation of a group of complex polysaccharides known as glycosaminoglycans. ${ }^{1}$ Intralysosomal accumulation of glycosaminoglycans results in progressive disability due to differing degrees of involvement of connective tissue and ground substance with predominant involvement of the skeletal system. Mucopolysacchariduria is a characteristic feature and detection of the specific glycosaminoglycan in the urine aids diagnosis. To date, eight separate types of mucopolysaccharidoses have been identified based on the specific lysosomal enzyme deficiency and each type has distinct clinical features leading to eponymous syndromes. Type IV mucopolysaccharidosis was described in 1929 by Morquio in Uruguay and Brailsford in England, and is due to defective catabolism of keratan sulphate and chondroitin sulphate. Two forms of the disease has been described. The classic form (type A) is due to deficiency of $\mathrm{N}$ acetylgalactosamine 6-sulphate sulphatase, and the mild form (type B) deficiency of $\beta$ galactosidase.

Patients with Morquio's syndrome have a typical appearance. Severe skeletal deformity due to spondyloepiphyseal dysplasia, appreci- able dwarfism with thoracolumbar gibbus, and pigeon chest are the main features. Joint laxity, mid face hypoplasia with wide spacing of teeth, and thinning of dental enamel are other characteristics. Corneal clouding and deafness develop in later life. Unusual among the mucopolysaccharidoses, intellect is normal or only minimally reduced. An invariable feature of Morquio's syndrome is the absence or severe hypoplasia of the odontoid process of the second cervical vertebrae. This requires posterior spinal fusion to prevent cervical myelopathy. Before development of adequate orthopaedic management of cervical spine subluxation, death resulted from progressive myelopathy by 20 to 40 years of age.

Cardiac involvement due to abnormal deposition of glycosaminoglycans has been described in most types of mucopolysaccharidoses, ${ }^{2}$ but little note made of abnormalities in Morquio's syndrome. The widely accepted association with Morquio's syndrome is aortic regurgitation. ${ }^{3}$ As there was no clinical evidence of aortic regurgitation in patients with mucopolysaccharidosis IV undergoing posterior spinal fusion in this centre, we decided to study a group of patients with the classical Morquio's syndrome echocardiographically in order to characterise their cardiac abnormalities.

\section{Patients and methods}

Ten patients with the classic or type A Morquio's syndrome attending the orthopaedic clinic at the Royal National Orthopaedic Hospital, London, were included in the study. The group had a median age of 12.5 years with a range of 3 to 40 years and comprised five male and five female patients. Biochemical confirmation of the diagnosis had been obtained in seven patients. Three of the older patients in whom biochemical data were not available (patients 8 , 9 , and 10 in the table 1 aged 30,38 , and 40 years respectively) had diagnostic clinical features.

Patients were evaluated clinically and echocardiographically with an Advanced Technical Laboratories (ATL) Mark 600 imager equipped with a mechanical sector scanner capable of pulsed Doppler echocardiography in addition to $M$ mode and cross sectional modes. Six months into the study we acquired an ATL Ultramark 6 ultrasound system with a phased array scanning head and patients with positive findings earlier in the study had echocardiography repeated using the new equipment with inclusion of colour Doppler imaging. Severe chest deformity necessitated unconventional echocardiographic views and patients were imaged in whichever position ensured adequate visualisation of car- 
Details of patients studied

\begin{tabular}{|c|c|c|c|}
\hline $\begin{array}{l}\text { Patient } \\
\text { No }\end{array}$ & $\begin{array}{l}\text { Age } \\
\text { (years), sex }\end{array}$ & Auscultation & Echocardiography \\
\hline $\begin{array}{l}1 \\
2 \\
3\end{array}$ & $\begin{array}{r}3, M \\
6, M \\
10, M\end{array}$ & $\begin{array}{l}\text { Normal } \\
\text { Normal } \\
\text { Soft ejection systolic murmur } \\
\text { at left sternal edge }\end{array}$ & $\begin{array}{l}\text { Normal } \\
\text { Normal } \\
\text { Aortic valve thickening; normal Doppler study }\end{array}$ \\
\hline 4 & $11, \mathrm{~F}$ & Soft systolic murmur at apex & $\begin{array}{l}\text { Elongated and thickened mitral valve with prolapse of both leaflets; } \\
\text { broad band mitral regurgitation on Doppler; aortic valve } \\
\text { thickening with mild aortic regurgitation }\end{array}$ \\
\hline 5 & $12, M$ & Normal & Normal \\
\hline 6 & & Normal & $\begin{array}{l}\text { Posterior mitral leaflet prolapse with narrow band mitral } \\
\text { regurgitation }\end{array}$ \\
\hline 7 & $14, F$ & $\begin{array}{l}\text { Soft ejection systolic murmur } \\
\text { at left sternal edge }\end{array}$ & Normal \\
\hline 8 & $30, M$ & Normal & $\begin{array}{l}\text { Posterior mitral leaflet prolapse; mild mitral regurgitation; bicuspid } \\
\text { aortic valve with normal function }\end{array}$ \\
\hline 9 & $38, F$ & $\begin{array}{l}\text { Ejection systolic murmur at left } \\
\text { sternal edge; loud S2 }\end{array}$ & $\begin{array}{l}\text { Thickened mitral leaflets with narrow band mitral regurgitation; } \\
\text { thickened aortic valve with mild aortic regurgitation; no aortic } \\
\text { valve gradient; left ventricle apical hypertrophy }\end{array}$ \\
\hline 10 & 40, F & Normal & $\begin{array}{l}\text { Pronounced thickening of mitral leaflets with mild stenosis (mean } \\
\text { gradient } 4-6 \mathrm{~mm} \mathrm{Hg);} \mathrm{mild} \mathrm{mitral} \mathrm{regurgitation} \mathrm{and} \mathrm{aortic} \\
\text { regurgitation; biventricular hypertrophy }\end{array}$ \\
\hline
\end{tabular}

diac structures. Images were obtained using $2 \cdot 5$ or $3.5 \mathrm{MHz}$ transducers depending on the patient's build and depth of structures to be imaged. All echocardiographic data were viewed and interpreted initially by one of the investigators (RMJ) and findings corroborated with observations made independently by one of the other investigators (RHS).

Echocardiographic mitral valve prolapse was defined as mid or late systolic posterior displacement of part of the mitral systolic closure line to at least $2 \mathrm{~mm}$ below the line joining the point of valve closure in systole (C) to the point of valve opening in diastole (D) with superior

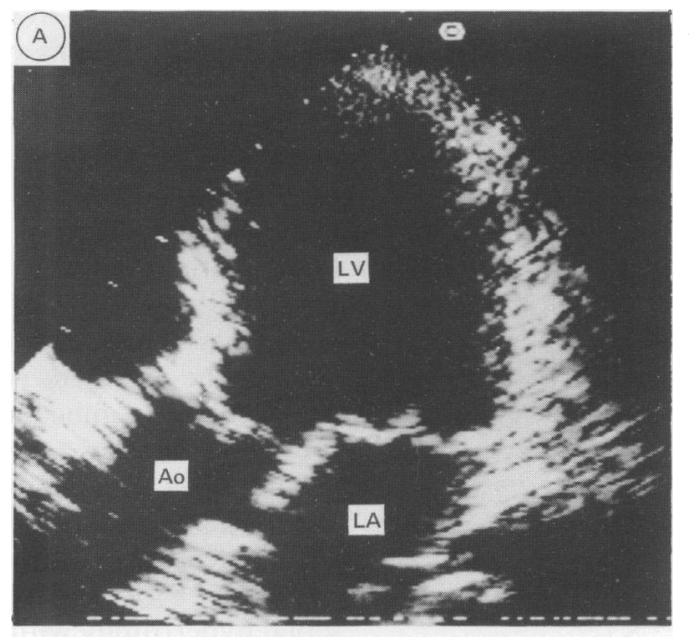

(B)

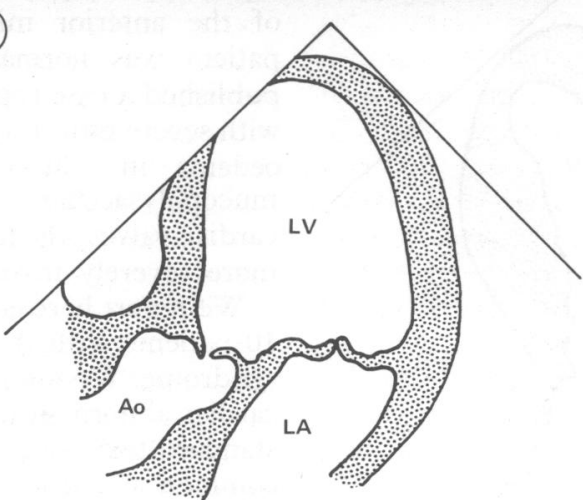

Figure 1 Systolic frame apical four chamber view of patient 4 showing mitral and aortic valve thickening and posterior bowing of both mitral leaflets in systole. $L V=l e f$ ventricle; $L A=$ left atrium; $A o=$ aorta. leaflet displacement confirmed on cross sectional echocardiography. It was also diagnosed if there was a pansystolic prolapse with posterior displacement with at least $3 \mathrm{~mm}$ below a line joining $\mathrm{C}$ and $\mathrm{D} .^{4}$

\section{Results}

During the year 1988, 10 patients known to suffer with the classic form of Morquio's syndrome were studied and the findings are summarised in the table. Within the limitations imposed by their skeletal deformities, none of the patients experienced cardiac symptoms.
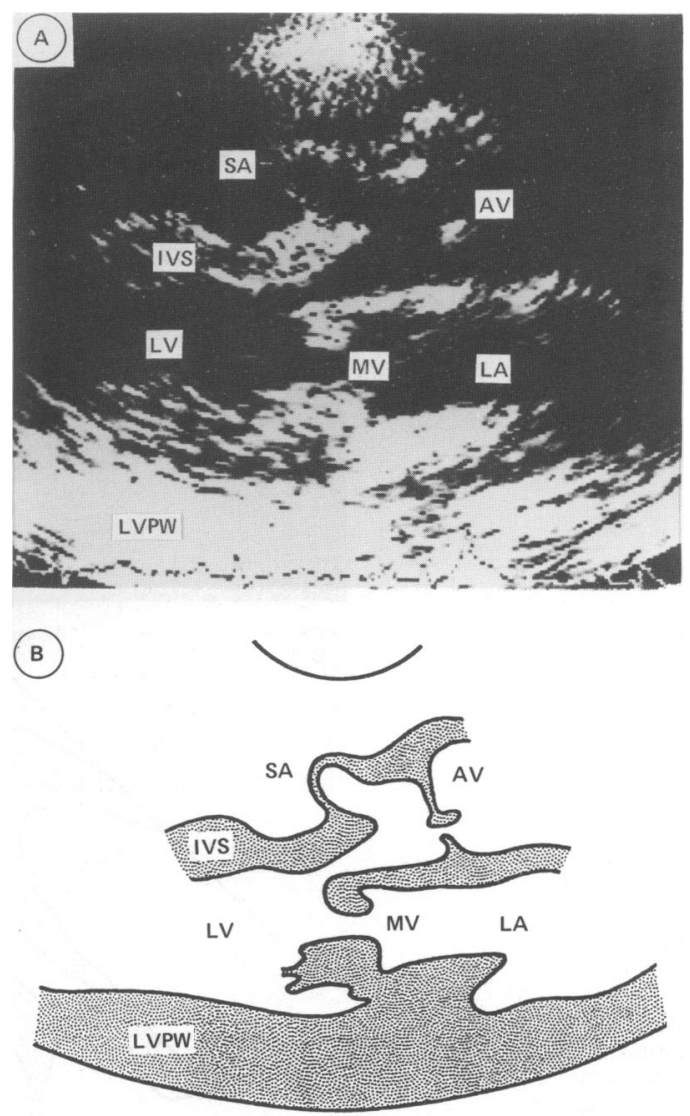

Figure 2 Diastolic frame parasternal long axis view of patient 10 demonstrating gross mitral leaflet thickening. There is aortic leaflet thickening to a lesser extent. An aneurysm of the membranous septum is also evident. $L V P W=$ left ventricular posterior wall; $M V=$ mitral valve; $A V=$ aortic valve; $I V S=$ interventricular septum;

$S A=$ septal aneurysm; $L A=$ left atrium; $L V=$ left ventricle 
No patient gave a history of rheumatic fever. All patients were in sinus rhythm and normotensive. Four patients had grade 2 systolic cardiac murmurs on auscultation three of whom had echocardiographic abnormalities. Three patients had echocardiographic valvular abnormalities without associated auscultatory findings. Overall, echocardiographic abnormalities were found in six $(60 \%)$ of those studied. These echocardiographic abnormalities are described in detail below.

\section{MITRAL VALVE}

Five $(50 \%)$ patients had mitral valve disease. Mitral regurgitation was easily detected on pulsed Doppler echocardiography in all of these, and clear systolic mitral leaflet prolapse was evident in three. The mitral regurgitation was haemodynamically mild in all except patient 4 in whom mild left ventricular and atrial dilatation was also present (fig 1). The oldest in the group (patient 10) had severe thickening of both mitral leaflets to the point of mitral stenosis (fig 2). Her mitral valve cross sectional planimetric area was $1.4 \mathrm{~cm}^{2}$ and mean gradient across the valve at rest was 4 to $6 \mathrm{~mm}$ Hg. Colour Doppler echocardiography demonstrated a left ventricular inflow jet of mixed velocities.
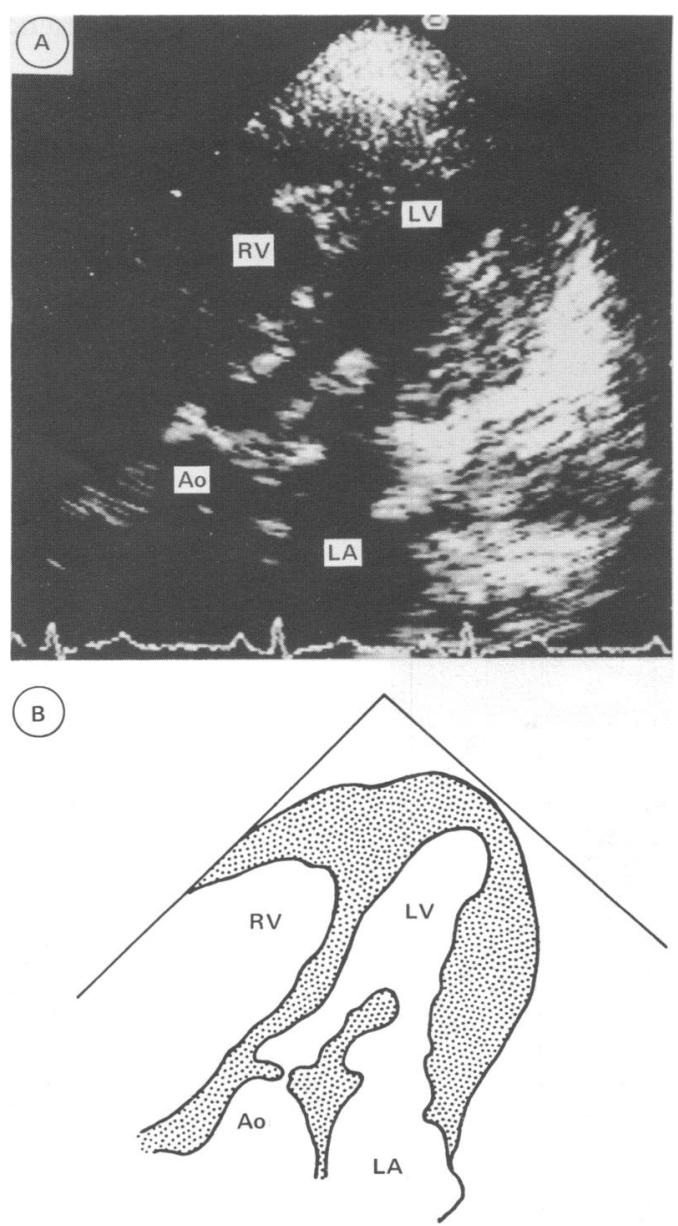

Figure 3 Diastolic frame apical view of patient 10 showing mitral leaflet thickening and hypertrophy of the left ventricular lateral wall. There is also right ventricular apical ventricular lateral wall. There is also right ventricular apical
thickening demonstrated on this frame. $R V=$ right ventricle; $L A=$ left atrium; $L V=$ left ventricle; $A o=$ aorta.
AORTIC VALVE

Aortic valve involvement was detected in four patients. In one (patient 3), this was an isolated abnormality with no functional sequelae. The other three patients had pulsed Doppler evidence of mild to moderate aortic regurgitation. Colour Doppler study showed mild turbulence of outflow through the aortic valve in two patients but in all the peak aortic flow velocity was less than $1.2 \mathrm{~m} / \mathrm{second}$. No patient had aortic stenosis.

\section{MYOCARDIAL INVOLVEMENT}

The two patients (9 and 10), who had both mitral and aortic valve disease, also had myocardial abnormalities. These were biventricular concentric hypertrophy in patient 10 (fig 3 ) and left ventricular apical hypertrophy in patient 9 . Pulsed Doppler trace on the left ventricular inflow in patient 10 showed a higher flow velocity during atrial systole which suggests a degree of restriction of left ventricular filling.

\section{OTHER ANOMALIES}

Patient 10 also had evidence of a membranous septal aneurysm (fig 2). Patient 8 had a bicuspid aortic valve but without valve stenosis or regurgitation.

\section{Discussion}

The reported frequency of Morquio's syndrome is 0.33 per 100000 live births. ${ }^{2}$ There are only 44 cases of Morquio's syndrome registered with the Society for Mucopolysaccharide Diseases in the United Kingdom. It is therefore, a very rare condition. Cardiovascular disease has been reported in all the mucopolysaccharidoses. Dawson commented in 1954 that it was unusual for a patient with mucopolysaccharidosis to have a microscopically normal heart at necropsy. ${ }^{5}$ There are many publications concerning the prevalence of cardiac disease in the more common mucopolysaccharidoses, but a survey of the literature reveals only two papers dealing with cardiac disease in the Morquio's syndrome. Gross et al reported echocardiographic features in three patients, one of whom had severe aortic regurgitation, another discrete septal hypertrophy with systolic anterior motion of the anterior mitral leaflet and the third patient was normal. ${ }^{6}$ Ireland and Rowlands published a case report of a 48 year old women with severe mitral stenosis leading to pulmonary oedema in whom necropsy showed severe mucopolysaccharide infiltration of all four cardiac valves, the left sided valves considerably more severely involved than the right. ${ }^{7}$

We report here echocardiographic features of 10 patients with the classic form of Morquio's syndrome. We found a high prevalence of silent cardiac abnormalities and contrary to reports in standard text books, mitral involvement was as common as aortic valve disease. In this study, mitral valve involvement was more severe with patient 10 being the extreme example. Mild to moderate mitral regurgitation with or without prolapse of one or both leaflets was the most 
frequent pattern. Aortic valve disease was comparatively mild in all four cases with cuspal thickening and mild to moderate aortic regurgitation detected only on Doppler echocardiography. Endocardial thickening also appears to be a late feature of the disease. This was initially commented upon by Gross et al where discreet septal hypertrophy was documented. ${ }^{6}$ In this study endocardial thickening was evident in two patients, the magnitude of which was out of proportion to their valvular abnormalities.

The pathology of cardiac involvement in the common mucopolysaccharidoses is well documented. Characteristically, swelling and vacuolation of cells and connective tissue of all layers of the heart is seen. There is only one paper documenting the cardiac pathology in Morquio's syndrome, and this described mucopolysaccharide infiltration of all four cardiac valves. ${ }^{7}$ Although histological data is not presented in this study, mucopolysaccharide accumulation and consequent collagen derangement is the probable cause of the valvular and endocardial abnormalities. The increasing frequency and severity of cardiac abnormalities with age found in this series is in keeping with this progressive pathological process.

Now that more of these patients are surviving into later life, cardiac abnormalities will assume a more prominent role in their prognosis. These cardiac abnormalities are not always evident clinically as exemplified by patient 10 who despite her quite severe cardiac involvement, demonstrated on echocardiography, had no clinical signs of cardiac disease. We suggest that these patients should have echocardiography as part of their routine assessment and follow up.

Although most of these patients will be poor candidates for major cardiac surgery, orthopaedic intervention will be necessary at some stage, usually in childhood. Haemodynamically their cardiac abnormalities are unlikely to be important in the context of surgery, but the high prevalence of valvular abnormalities found in this study suggests that antibiotic prophylaxis against infective endocarditis is indicated.

We would like to thank Mrs Mary Toole of the Society for Mucopolysaccharide disease for her most gracious assistance in recruiting patients for this study.

This work was in part supported by the Middlesex Hospital and Medical School Research Fund.

1 McKusic VA, Neufeld EF. The mucopolysaccharide storage diseases. In: Stanbury JB, Wyngaarden JB, Fredrickson inherited diseases. 5th Ed. New York: McGraw Hill, 1983: 751-77.

2 Lowden AJ, Pearse RG, Rowe RD. Cardiac involvement in the mucopolysaccharidoses. In: Keith JD, Rowe RD, the mucopolysaccharidoses. In: Keith JD, Rowe RD, Vlad P, eds. Heart disease in infancy and chid
New York: MacMillan, 1978:995-1003.

3 McKusic VA, Kaplan D, Wise D, et al. The genetic mucoMcKusic VA, Kaplan D, Wise D, et al. The g
polysaccharidoses. Medicine 1965;44:445-83.

4 Wilcken DE, Hickey AJ. Lifetime risk for patients with mitral valve prolapse of developing severe valve regurgitation requiring surgery. Circulation 1988;78:10-4.

5 Dawson IMP. The histology and histochemistry of gargoylism. foumal of Pathology and Bacteriology 1954;67: 587-604.

6 Gross DM, Williams JC, Caprolic C, Dominguez B, Howell RR. Echocardiographic abnormalities in the mucopolysaccharide storage diseases. Am J Cardiol 1988;61:170-6.

7 Ireland MA, Rowlands DB. Mucopolysaccharidoses type IV as a cause of mitral stenosis in an adult. Br Heart $\mathcal{F}$ 1981;46:
$113-5$. 